\title{
FRAUENROLLEN UND POPMUSIK IN DER ARABISCHEN WELT: VON UNANTASTBAREN STARSÄNGERINNEN, MODELS IN UNTERWÄSCHE UND SELBSTBEWUSSTEN MUSIKERINNEN
}

\section{Thomas Burkhalter}

Schaut man heute arabisches Satellitenfernsehen, so scheint sowohl für arabische wie auch westliche Musikfreunde und Intellektuelle alles klar zu sein: Die arabische Welt befindet sich im Niedergang. Wo früher begnadete Sängerinnen wie Umm Kulthum und Fairuz auftraten, schwenken heute Tausende von Popsternchen ihre Hüften. Wo früher mehr oder weniger gehaltvolle ägyptische Filme gezeigt wurden, läuft heute bloß noch Schrott. Die Frauen- und Männerrollen scheinen klar verteilt. Die Männer dürfen Machos und Softies sein, die Frauen sind vor allem eines: Sexobjekte.

\section{Kontext: Die Veränderungen in der arabischen Popmusik-Welt}

Die arabische Medienwelt hat sich vor allem ab den 1990er Jahren radikal verändert. Bis dahin waren Ägypten und Libanon die Medienmetropolen der arabischen Welt, heute ist es Saudi-Arabien. 1994 startete der saudische Prinz und Geschäftsmann Al-Waleed bin Talal sein Unterhaltungsnetzwerk $A R T$ in Rom. ART setzte auf ein westlich gestyltes Unterhaltungsprogramm mit Hollywood-Filmen, US-amerikanischen Seifenopern und Talkshows. Andrew Hammond (2007) spricht von einem radikalen Wandel in der arabischen Welt: Der ägyptische Präsident »Abdel-Nasser wollte die Bevölkerung auf der Straße, die Saudis wollen die Bevölkerung auf dem Sofa«. Die Veränderungen waren riesig. Arabisten und Medienwissenschaftler (Hammond 2007; Miller 2007; Sakr 2001, 2007) argumentieren, dass die arabische Welt zum ersten Mal überhaupt ein panarabisches Programm konsumiert - von Casablanca bis Dubai. Jeder und jede schaut sie sich an: die panarabischen 
Popstars; die islamischen Televangelisten; den Ägypter Shaaban Adel Rahim, der gegen Israel und die USA wettert; und Sami Yusuf, der Gott in kitschigen Liedern auf Englisch preist.

Die Popmusik-Industrie ist im Zuge dieser Veränderungen zu einer Videoclip Industrie geworden. Die Satelliten-TV-Stationen investierten in die Produktion von Popmusik-Videos und starteten Musikkanäle - ähnlich wie MTV oder VIVA. Der Rotana-Sender von Bin Talal nimmt heute beinahe eine Monopolstellung ein. Rotana besitzt Produktionsstudios in Beirut, Dubai und Kairo und kontrolliert beinahe den gesamten arabischen Musikmarkt. Rotana besitzt auch den größten Teil aller ägyptischen Filme - sie kamen durch geschickte und aggressive Firmenübernahmen in den Besitz des saudischen Geschäftsmanns. Die anderen Popmusiklabels kämpfen neben Rotana ums nackte Überleben. Einige sind auf die Produktion von religiöser Musik umgesattelt - eine interessante Gegenstrategie (Said 2004). Die Formel ist klar: Die Finanzen kommen aus Saudi Arabien, Dubai dient als das wirtschaftliche Zentrum, Beirut und Kairo dienen als Produktionsstätten: von hier kommen die meisten Songschreiber, die Sängerinnen, die Texter und die Arrangeure - alles eigene Geschäftszweige. Ein libanesischer Musiker erklärte mir, wie das Popgeschäft läuft:

"Normally you have the singer and his or her manager. They go to a lyricist, then to a composer, and then to an arranger. At each person they pay a certain amount of money. The melody, mostly written by an Egyptian, costs between $\$ 3,000$ and $\$ 3,500$; the lyrics cost $\$ 1,000$ to $\$ 2,000$; the arrangements $\$ 3,000$ to $\$ 8,000 \ll$ (Fadel 2005).

Die Popstars verdienen sehr gut, wenn sie denn in den Pop-Olymp vorstoßen. Der ägyptische Popstar Amr Diab soll pro Album fünf Millionen Dollar verdienen. Nawal al-Zoughbi, eine libanesische Starsängerin 900.000 Dollar (Ismail 2005). 2001 habe ich Nawal al Zoughbi mit dem Auftrag, eine arabische Popsängerin für ein Openair-Festival in der Schweiz zu gewinnen, in ihrer Villa besucht. Der Manager wollte 40.000 Dollar für ein Konzert. Verhandeln schien ausgeschlossen. Europa ist nicht der Primärmarkt. Diese Sängerinnen (und ihre Manager) verdienen ihr Geld bei Konzerten und Privatfesten im Golf. Manchmal singen sie an sechs Orten in einer Nacht: an privaten Parties, in Hotels und in TV Shows. Zwanzig Minuten pro Show, das ergibt 100.000 Dollar und mehr pro Nacht. Und Hubert Ghorayeb von EMI Lebanon fügt hinzu: Frauen haben die größeren Chancen in diesem Geschäft. Sie sind allerdings durch rigide Verträge an Rotana gebunden (Hackensberger 2005). Rotana verfügt über alles: Auftritte, Konzerte, CDs und Videoclips - ein 
$»$ Rundum «-Vertrag quasi. Letztere Praxis ist allerdings schon seit dem VorSatelliten-Zeitalter bekannt, wie mir ein libanesischer Musiker erklärte:

»Music in Lebanon was always controlled and manipulated by certain people: First by the Rahbani clan, today by Rotana, or World Music producers like Michel Elefteriades. The Rahbanis signed contracts with every talented singer in the country and let them sing background vocals - or let them just disappear. And they keep saying that they created Lebanese music, or they brought rock to Lebanon (Ghassan Rahbani), or created Lebanese Bossa Nova (Ziyad Rahbani). I just hate them for that, and I would never work with them again« (Anonym 2005).

Will Rotana andere Mediensender schwächen, so kauft Bin Talal auch die Sängerinnen der Konkurrenz - und das ohne eigentlichen Geschäftsplan für die betreffende Künstlerin. Es kursieren viele Anekdoten von Gerichtsfällen, in denen sich Sängerinnen von Rotana frei feilschen wollten. Und dann ist da das hartnäckigste Gerücht überhaupt: Die weiblichen Popstars arbeiteten sich hoch, indem sie sich bei Scheichs, Königen, Politikern und Geschäftsmännern prostituierten - das behaupten viele, Beweise dafür gibt es freilich nicht. Eine libanesische Sängerin sagte es mir so:

»The managers talk very badly and nastily about their stars, and they treat the women singers like prostitutes, or like animals. Many of the main men in this business make stars out of their girlfriends. Normally they give them contracts for fifteen years, so when love is over, the career is over as well« (ebd.).

Alle diese Anschuldigungen schienen sich zu bestätigen, als im Juli 2008 die libanesische Popsängerin Suzanne Tamim ermordet in ihrem Appartement in Dubai aufgefunden wurde. Ihr Liebhaber, ein ägyptischer Geschäftsmann und enger Berater vom ägyptischen Präsidenten Hosni Mubarak, soll einen Auftragsmörder angeheuert haben: CNN berichtete ausführlich und sprach von großen Prostitutionsringen, in die Popsängerinnen, Geschäftsmänner und Politiker verstrickt seien.

»Rumors abound of businessmen and politicians peddling out actresses and singers in prostitution rings. The frequent marriages and divorces of celebrities and businessmen make big news. [...] People in the Arab world have long followed with fascination and moral clucking the tales of businessmen and politicians cavorting with actresses, belly-dancers and singers - a sort of Hollywood Babylon in the conservative Muslim Middle East« (Nasr 2008).

Im Mai 2009 wurde der ägyptische Geschäftsmann in Kairo zum Tode verurteilt. Seine Anwälte haben Berufung eingelegt. 


\section{Videoclips}

Im Folgenden möchte ich auf diese omnipräsenten arabischen Videoclips, ihre Inhalte und Ästhetik eingehen. Zunächst einmal fällt eines auf: Die Gesichter wechseln ständig: George Wassouf, Najwa Karam und Nawal alZoughbi, die Stars Anfang der 2000er Jahre, wurden ersetzt durch Haifa Wehbe und Nancy Ajram. Andere neue Gesichter erscheinen auf den Videoclip-Sendern fast täglich. Früher sang eine Sängerin im Dialekt ihres Heimatlandes. Heute singt sie ein Lied im libanesischen Dialekt, eines auf Ägyptisch, eines im Golf-Dialekt - man will möglichst alle Geschmäcker bedienen in der arabischen Welt. Tony Sfeir vom Beiruter Musikladen $C D$ Thèque findet es einfach nur schlimm:

"You find a girl, let her undergo 27 operations, and she becomes beautiful on TV. You tell her to sing, and she sings, you give her Egyptian songs, and she sings them, then songs from Turkey, and she sings them, music from Spain, Greece, all mixed together. It becomes music without an identity, sung by a singer who lost her identity« (Sfeir 2005).

Viele Videoclips werden heute außerhalb der arabischen Welt gedreht. Vor allem in der Türkei und in Osteuropa finden die Regisseure für wenig Geld Models mit dem beliebten hellen Hauttyp. In einem Videoclip soll Miss Kasachstan für 100 Dollar aufgetreten sein. Drehen im Ausland - das zeigt auch: Ich bin erfolgreich und weltoffen (Ismail 2005).

Vor ein paar Jahren waren die Videoclips noch billig und einfach produziert, heute sind sie sehr professionell. Die teuersten Clips kosten bis zu 300.000 Dollar, billigere um die 20.000 Dollar und Low Budget-Produktionen 3000 bis 5000 Dollar (ebd.). Früher wanderten die Sängerinnen und Sänger in billigen Kulissen voller Palmen und kitschiger Wasserfontänen herum. Heute trinken sie in einer Stadt italienischen Espresso oder flirten in einem idealisierten Dorf mit ungehobelten Farmern. Die Männer sind oft David Beckham-Typen oder Latin Lovers. Sie tragen gerne Designer-Kleidung, oder sie zeigen ihre gestählten Oberkörper. Frauen und Männer fahren in teuren Autos herum, oder sie tanzen an perfekten Stränden. Die Frauen räkeln sich gerne auf einem luxuriösen Bett in einer luxuriösen Villa. Sie tragen mal Lingerie, mal treten sie als orientalische Schönheiten auf.

90\% der Lieder und Videoclips handeln von Liebe und Sehnsucht, 10\% von Hochzeiten, dem Mutter-Sein und von Kindern. Viele der Videos provozieren immer wieder konservative und religiöse Kreise. Die Satellitensender sind aber zu einem großen Teil immun gegenüber dieser Kritik. Sie senden 
oft aus Europa und den USA und lassen sich nicht einfach zensieren. Gelegentlich aber wird der Druck derart groß, dass ein Video tatsächlich nicht mehr gespielt wird. Für Skandale sorgten etwa die Clips von Ruby aus Ägypten. In der kommerziellen Popmusikwelt ist sie die Königin des Bauchtanzes. Sie wackelt ihre Hüften zu Techno-Pop und zu lokalem Chaabi-Pop. Und die in verschiedenen Kontexten und verschiedenen Kleidern: Mal als Cheerleader, dann im Gymnastikanzug, dann im eleganten roten Kleid, im Strandbikini, an der Stange im Striplokal oder im Stil von Cleopatra mit einer Boa um den Hals (Comer 2005).

Oft führen die Debatten um die skandalösen Videos aber bloß dazu, dass die Sängerinnen noch berühmter werden. Einige Videos provozieren muslimische Traditionen ganz direkt: Darrah (2008) berichtet von Samars »Ama Ach-kta«: Im Video sieht man sie in einem typischen maghrebinischen Hochzeitsdress und mit der traditionellen Henna-Bemalung auf den Fingern. Sie singt davon, wie sehr sie sich auf die Hochzeitsnacht freut.

\section{Haifa Wehbe}

Haifa Wehbe ist derzeit die wichtigste libanesische Sängerin im panarabischen Popmusikgeschäft. Sie ist shiitische Muslimin aus dem Südlibanon und hat mit ihrem Ehemann in Afrika gelebt. Laut Gerüchten floh sie in den Libanon, weil er sie geschlagen hatte. Ihre gemeinsame Tochter musste sie zurücklassen. Im Libanon vergleichen einige Intellektuelle und SubkulturKünstler Haifa bereits mit Marilyn Monroe oder Brigitte Bardot.

Berühmt ist Haifa vor allem durch ihre Sexyness und ihre anzüglichen Videoclips. Im Clip »Mosh Adra Astana« gleitet sie im Badeanzug aus einem Swimming Pool. Dazu erklingt ein Gitarrenriff, eine hart geschlagene Darabukka-Trommel und ein intensiver elektronischer Sound. Die chaotische Musik suggeriert wohl: Haifa ist aufgewühlt. Der Liedtext bestätigt das:

»Ich kann keinen Tag mehr warten.

Komm zurück. Ich flehe dich an, sehnsüchtig.

Mein Liebling, Traum meines Lebens, ich will dich sehen.

$\mathrm{Du}$, der du dich entziehst, ich habe Zeit.

Mein Liebling, das ist der letzte aller Seufzer.

Wir werden hier zusammenkommen.

Viel zu lange weg warst du für mich.

Für zu viele Jahre habe ich ohne Komfort und Sicherheit gelebt.«

(Übersetzung T.B.) 


\section{Kultureller Niedergang}

In Syrien, so Jonathan Shannon, hätten die meisten Popsängerinnen einen Mangel an Ernsthaftigkeit und Aufrichtigkeit - »lack sincerity (sidq)« - , und sie seien darum auch keine Künstlerinnen. Ein Musikliebhaber erzählte Shannon Folgendes: »Modern singers may sing of love, but they don't know real love - they are mimicking true love, and at worst fabricating the experience; theirs is false love (Shannon 2006: 155). Andere meinten: »They sing of love but are really only interested in sex« (ebd.). Die subkulturellen Musikszenen in der arabischen Welt sind einfach bloß genervt und wütend. Zum Beispiel der Rapper O-Marz aus Beirut:

»Haifa Wehbe, George Wassouf, what the fuck is that! I don't care how much he loves his girls and how much she longs for her beloved! If we had Umm Kulthoum back, I would be the happiest man on earth! It's a fucking conspiracy! The political leaders and the big businessmen are happy when the crowd consumes this bullshit and stays stupid « (O-Marz 2005).

Die Rap-Gruppe Aks'ser hat mit dem Videoclip »Ana Mich Illik« (2005) eine Parodie auf die Popmusik-Industrie produziert. Wir sehen die Rapper durch die Straßen Beiruts rennen, verfolgt von leicht bekleideten libanesischen Schönheiten (Burkhalter 2011). Der Rocker Gassan Rahbani widmet dem panarabischen Popmarkt ein ganzes Lied. Die Lyrics von »Everybody's Sick But Me sind im Booklet seiner CD Braindamage (2009) nachzulesen:

»Pathetic thoughts; disgusting plans, frantic lives; dark nights; ruling egos, ignorance is knowledge; killing is needed; praying is a shame, worship cruelty; stealing respected; sacred greed; minds rotted; education raped; rights violated; love smashed; principles polluted; respect destroyed; ethics corrupted; purity affected; power is weakness; nastiness welcomed; malice is adored; betray is a goal; innocence is annoying; sense is frustrating; honesty is revolting; praying is shame [...]. Believe me, my friend, they think like this!«

Für weite Teile der Bevölkerung ist klar: Die panarabische Popmusik ist dekadent, billig und geschmacklos. Der Dichter Mahmoud Darwish (1995: 140) nennt das, was die Satellitenstationen offerieren, »Petro-Kultur «, sie habe den kulturellen Niedergang in der arabischen Welt zusätzlich beschleunigt. Für den Medienwissenschaftler und Ägypten-Experten Walter Armbrust gehen diese Argumente viel zu weit. Er zeigt auf, dass Sex nicht nur in den heutigen Videoclips wichtig ist, sondern in der gesamten Geschichte der arabischen Popkultur im 20. Jahrhundert: 
»Even the rhetoric of dismissing these video clips as vulgar and cheap resonates with the past one hundred years of Egyptian history. [...] It is crucial to recognize that some of these ideas have historical roots. One must be on guard against overstating the novelty of new media« (Armbrust 2005).

\section{Rückblick in die Geschichte der arabischen Popularmusik}

Die Diskussionen um Frauenrollen haben tatsächlich eine lange Vorgeschichte in der arabischen Welt. Der Musikethnologe Jihad Racy entdeckt eine klare Männerdominanz in der sogenannten Tarab-Kultur - der arabischen Kunstmusik der kleinen takht-Ensembles. Es gab in dieser Tarab-Kultur aber auch immer wieder große Sängerinnen. Racy betont, dass gerade Sängerinnen der Musik eine besondere Note geben können:

»Female singing is recognized as an effective conveyor of musical ecstasy. In various historical epochs, male audiences have marvelled at women who sing, partly because the ecstasy conveyed by their voice was supposedly reinforced by their physical appearance« (Racy 2003: 17).

Und dennoch: Die Position von Sängerinnen wurde immer wieder von konservativen Attitüden bedrängt. Es gibt einen weit verbreiteten Glauben, dass eine professionelle Sängerin ihren Ehe- und Mutterpflichten nicht korrekt nachkommen könne. Fairuz wurde auch deshalb die populärste Sängerin des Libanons, weil sie es immer wieder geschafft hat, die Rollen der Künstlerin, Mutter und Ehefrau zu verbinden (Stone 2008; Habib 2005; Asmar 2002; Asmar/Hood 2001). In ihren Musiktheaterstücken spielte Fairuz das unschuldige Mädchen vom Lande, in ihrem Privatleben ging sie nie auf Parties und gab keine Interviews: Sie baute sich eine Reputation wie die der Heiligen Jungfrau Maria auf, wie Ines Weinrich (2006: 142f., 341, 407) schreibt. Sabah, ihre große Konkurrentin, war das pure Gegenteil. Sabah war Stammgast bei allen Parties, sie sorgte immer wieder für Skandale. Dass sie nie den gleichen Status erreichte wie Fairuz, hat wahrscheinlich auch damit zu tun.

Die ägyptische Diva Umm Kulthum trat zuerst als Knabe auf (Danielson 1997; Gsell 1999). Später wurde sie dann die wichtigste arabische Sängerin. Sie war aber immer geschützt durch hohe ägyptische Politiker.

»Umm Kulthum was a model of female decency. As a woman, her conservative religious background, and the full protection of her own family all accorded her great respect. [...] When she stood up to sing, there was in front of 
her an invisible sign that read Do Not Touch. She was learned and cultured, refined and faithful to her art« (Racy 2003: 37).

Asmahan war eine von Umm Kulthums schärfsten Konkurrentinnen. Sie war eine unabhängige, starke Selfmade-Frau, wie Sherifa Zuhur (2001: 143-164) schreibt. Asmahan starb an einem mysteriösen Autounfall. Sie wurde umgebracht, glauben viele bis heute.

Tanzen auf der Bühne oder nicht, das scheint bis heute ein großes Thema: Außerhalb der Popmusikindustrie stehen die arabischen Sängerinnen meist unbeweglich auf der Bühne. Ich erlebte zum Beispiel die christlich libanesische Protestsängerin Julia Boutros am Internationalen Festival von Beiteddine. Sie sang ihre patriotischen Lieder für den Süden Libanons mit größter Leidenschaft und sie stand auf der Bühne, fest verankert, wie ein Fels. Sie verzog keine Miene, selbst als das Publikum frenetisch applaudierte.

\section{Die subkulturellen Musikszenen}

Musikerinnen und Sängerinnen der aufstrebenden Subkultur-Szenen suchen nach neuen Wegen. Sie ziehen Asmahan und Sabah, Umm Kulthum und Fairuz vor. Sie sind selbstbewusst und wollen sich nicht durch die konservative Umgebung einschüchtern lassen. Frauen sind Musikerinnen, Sängerinnen, Tonmeisterinnen, Künstlerinnen, DJs und Rapperinnen. Sie musizieren mit Männern, die mit der Macho-Gesellschaft nichts mehr zu tun haben wollen - das sagen sie jedenfalls (Burkhalter 2007). Diese subkulturellen Szenen werden finanziell und informell gerne von internationalen Medienstationen und NGOs unterstützt - von MTV, der Heinrich Böll-Stiftung, dem Goethe-Institut. Die Sängerinnen und Musikerinnen werden als Stimmen für Frauenrechte eingesetzt; manchmal möchten sie aber einfach nur Künstlerinnen sein (Burkhalter 2011).

Gender-Fragen sind aber auch innerhalb der Subkultur-Szene weiterhin aktuell. "Sometimes, the Lebanese journalists ask me why I write such complex, >masculine < music «, hat mir Joelle Khoury (2005) in einem unserer Interviews erzählt, »they look at me, a feminine looking woman, thin, and a mother. And they ask, if I want to prove anything «. Khoury argumentiert, dass es nicht einfach sei als Musikerin, selbst im kleinen alternativen Musikerzirkel:

»Even my band sometimes has difficulties in accepting me, the woman, as the bandleader. Even though we are like a family and we love each other, I feel 
that the musicians sometimes have a problem with the fact that they play the music composed by me, a woman - even my husband. They make stupid jokes, and they are nagging all the time: Why do your write such complicated music? Do you want to pretend to be a great pianist, or a smart woman? Or do you just want to be different? «(Khoury 2005).

Joelle Khoury hat Strategien gefunden, wie sie diese Hürden überwinden kann. Manchmal sagt sie einfach ihrem Mann Maurice, er solle doch mit den Musikern sprechen: »They do listen to my advice coming through my husband, but they do not like my direct advice too much« (ebd.).

2005 hat Joelle Khoury eine Komposition für das libanesische Symphonieorchester geschrieben. Neue Musik - ziemlich ungewöhnlich für das Orchester. Das Stück wurde dann bloß einmal kurz geprobt und im Konzert miserabel gespielt. Raed El Khazen, ein Jazzgitarrist, hat das Konzert gesehen: Ein riesiger Skandal, sagte er mir, bei einem Mann wäre das nicht passiert:

»Many male musicians here think that Joelle is just a crazy woman: If you listen to her music, you know that she is one of the most serious, and most professional musicians working here. But still, I keep hearing jokes that she is a woman in a crisis, in her menopause, or whatever« (Khazen 2005).

Das alles hat zu tun damit, dass der Libanon letztlich eine sehr konservative Gesellschaft ist - und zwar die christliche und die muslimische Gemeinschaft zu gleichen Teilen, wie Khoury findet:

»As a woman you have to be nice to the men, you have to admire them and make them feel that they are intelligent, important, or even heroes. [...] If they feel that a woman thinks differently, and is critical about an issue, they get annoyed. Men have the right to be critical about things, women do not« (Khoury 2005).

Khoury aber will ihr Leben aktiv definieren: »I don't want to accept the role we women have in this society: Always waiting, for the husband, for the boyfriend, for your child, for better times« (ebd.). Joelle Khourys großes Vorbild ist die Schriftstellerin Virginia Wolf, laut Khoury eine starke, eigenwillige, unabhängige Frau (Burkhalter 2011).

Die Sängerin Yasemine Hamdan verkörpert ein ähnliches Frauenbild. Sie arbeitet heute im Duo Y.A.S mit Mirwais Ahmadzaï, der unter anderem die Alben Music und American Life von Madonna produziert hat. Hamdan ist selbstbewusst, stark, sexy. Ihr Videoclip »Get It Right« spielt in Kairo, im Jahr 2019. Yasemine Hamdan fliegt als erste arabische Astronautin zum Mond. Man spürt förmlich ihren Traum: von einer modernen, weltoffenen 
arabischen Welt und von einer selbstbewussten, erfolgreichen arabischen Frau.

\section{Literatur}

Armbrust, Walter (2005). »What Would Sayyid Qutb Say? Some Reflections on Video Clips.« In: TBS. Transnational Broadcasting Studies, Nr. 14 (Spring). Online unter: http://www.tbsjournal.com/Archives/Spring05/armbrust.html (Zugriff am 26.5.2010).

Asmar, Sami (2002). »Fairouz: a Voice, a Star, a Mystery.«In: Al Jadid. A Review \& Record of Arab Culturesand Arts 8, Nr. 38 (Winter). Online unter: http://www. aljadid.com/music/fairouza_voice_star_mystery.html (Zugriff am 26.5.2010).

Asmar, Sami / Hood, Kathleen (2001). »Modern Arab Music: Portraits of Enchantment from the Middle Generation. «In: Colors of Enchantment. Theater, Dance, Music, and the Visual Arts of the Middle East. Hg. v. Sherifa Zuhur. Cairo, New York: The American University in Cairo Press, S. 297-320.

Burkhalter, Thomas (2007). »Mapping Out the Sound Memory of Beirut: A Survey of the Music of a War Generation. "In: Itinéraires esthétiques et scènes culturelles au Proche-Orient. Hg. v. Nicolas Puig und Franck Mermier. Beirut: Institut Francais du Proche-Orient, S. 103-125.

Burkhalter, Thomas (2011). Creating Sense out of Chaos: New Sounds from Beirut (noch nicht erschienen).

Comer, Brooke (2005). »Ruby: The Making of a Star.« In: TBS. Transnational Broadcasting Studies, Nr. 14 (Spring). Online unter: http://www.tbsjournal. com/Archives/Spring05/comer.html (Zugriff am 26.5.2010)

Danielson, Virginia (1997). The Voice of Egypt. Umm Kulthum, Arabic Song, And Egyptian Society in the Twentieth Century. Chicago, London: The University of Chicago Press.

Darrah, Usahma Felix (2008). »Arabiens Jugend tanzt sich in die Moderne. Der Erfolg der Pop-Videos als Spiegel einer Kulturrevolution.« In: Neue Zürcher Zeitung vom 8. August. Online unter: http://www.nzz.ch/nachrichten/kultur/ medien/arabiens_jugend_tanzt_sich_in_die_moderne_1.801135.html (Zugriff am 26.5.2010).

Darwish, Mahmoud (1995). Memory for Forgetfulness - August, Beirut, 1982. Translated from the Arabic by Ibrahim Muhawi. Los Angeles: University of California Press.

Gsell, Stefanie (1999). Umm Kulthum. Persönlichkeit und Faszination der ägyptischen Sängerin. Berlin: Das Arabische Buch.

Habib, Ken (2005). The Superstar Singer Fairouz and the Ingenious Rahbani Composers: Lebanon Sounding. Santa Barbara: University of California (unveröffentl. Thesis).

Hackensberger, Alfred (2005). »Popkultur in der arabischen Welt.«In: Telepolis, 19. Oktober, http://www.heise.de/tp/r4/artikel/20/20976/1.html (letzter Zugriff 26.5.2010).

Hammond, Andrew (2007). "Saudi Arabia's Media Empire: Keeping the Masses at Home.« In: Arab Media \& Society 3, Fall. Online unter: http://arabmedia society.sqgd.co.uk/?article $=420$ (Zugriff am 26.5.2010). 
Ismail, Mohamed Hossam (2005). The Closed Circly vs. the Discontinuity of the Self: The Influences of Globalization upon Contemporary Arab Music Videos. Paper, präsentiert auf der International Conference of the IAMCR »Media Panics: Freedom, Control and Democracy in the Age of Globalization«, Taipei, Taiwan Juli 26-28.2005; unveröffentlicht.

Lagrange, Frederic (2000). Al-Tarab. Die Musik Ägyptens. Heidelberg: Palmyra.

Miller, Flagg (2007). The Moral Resonance of Arab Media: Audiocassette Poetry and Culture in Yemen. London: Cambridge University Press.

Racy, Ali Jihad (2003). Making Music in the Arab World, The Culture and Artistry of Tarab. Cambridge: Cambridge University Press.

Said, Summer (2004). »Artists' Exodus Sparks Fear of Culture Decline.«In: Business Monthly, August. Online unter: http://www.amcham.org.eg/resources_publicat ions/publications/business_monthly/issue. asp?sec=15\&im=8\&iy=2004 (Zugriff am 26.5.2010).

Sakr, Naomi (2001). Satellite Realms: Transnational Television, Globalisation \& the Middle East. London: I.B. Tauris Publishers.

Sakr, Naomi (2007). Arab Television Today. Arab Media and Political Renewal: Community, Legitimacy and Public Life. London: I.B. Tauris Publishers.

Shannon, Jonathan Holt (2006). Among the Jasmine Trees. Music and Modernity in Contemporary Syria. Middleton: Wesleyan University Press.

Stone, Christopher (2008). Popular Culture and Nationalism in Lebanon. The Fairouz and Rahbani Nation. New York: Routledge.

Touma, Habib Hassan (1998). Die Musik der Araber. Wilhelmshafen: Noetzel (erw. Neuausgabe).

Weinrich, Ines (2006). Fairuz und die Brüder Rahbani. Musik, Moderne und Nation im Libanon. Würzburg: Ergon.

Zuhur, Sherifa (2001). Asmahan's Secrets. Woman, War, and Song. London: Saqi Books.

\section{Interviews}

Anonym (2005). Interview mit dem Autor am 10. August in Beirut. Fadel, Michel (2005). Interview mit dem Autor am 18. August in Beirut. Khazen, Raed el (2005). Interview mit dem Autor am 7. Juli in Beirut. Khoury, Joelle (2005). Interview mit dem Autor am 25. Juli in Beirut. O-Marz (2005). Interview mit dem Autor am 9. September in Beirut. Rahbani, Ghassan (2006). Interview mit dem Autor am 9. Oktober in Beirut. Sfeir, Tony (2005). Interview mit dem Autor am 14. September in Beirut.

\section{Diskographie}

Aks'ser (2006). Aks'ser. EMI Music Arabia 09463473872 (Libanon). Eid, Rana (2003). ShShSh. La CD-Thèque (Libanon). Jubran, Kamilya / Hasler, Werner (2005). Wameedd. Unit Records W\&K 001 (Schweiz). 
Khoury, Joelle (2008). Dream She Is - Arabic Opera Monodrama For Woman's Voice.

Eka3 (Libanon).

Khoury, Joelle (2004). In-Version. Self Published (Libanon).

Khoury, Joelle (2003). Music \& Poetry. La CD-Thèque (Libanon).

Rahbani, Gassan (2009). Braindamage. Self Published (Libanon).

Y.A.S. (2009). Arabology. Opendisc 5317331 (Frankreich).

\title{
Videographie
}

Aks'ser (2005). »Ana Mich Illik«, http://www.myvideo.de/watch/7265410/Aks_ser_ Ana_Mich_Illik (Zugriff am 26.5.2010).

Nasr, Octavia (2008). Lebanese Pop Star Killed [CNN-Video vom 6. September], http://edition.cnn.com/video/\#/video/world/2008/09/06/nasr.labanese.star.ki lled.cnn?iref=allsearch (Zugriff am 26.5.2010).

Samar (2006). »Ama Ach-kta«, http://www.youtube.com/watch?v=xZEu4RyTNOU (Zugriff am 9.6.2010).

Whebe, Haifa (2007). »Mosh Adra Astana«, http://www.youtube.com/watch?v= xTdpiP7z4cl (Zugriff am 26.5.2010).

Y.A.S. (2009). "Get It Right«, http://www.youtube.com/watch?v=Bgl_oi3GV0Q (Zugriff am 26.5.2010).

\begin{abstract}
This article offers a survey of the discrepancies and transformations of gender roles of female musicians and singers in the Arab World. It starts with a short overview about female singers in the mass mediated pan Arabic pop music market. Then it looks back into the history of popular music in the Arab world: Star singers like Umm Kulthum and Fairuz became regional heroes not just because of their artistic gifts but because politicians and sometimes their husbands protected them. Women artists in today's subcultural music scene follow a different strategy: They want to be recognized as singers and musicians locally and globally. They are self-confident strong women who are not afraid to bring out their sexiness.
\end{abstract}

